\title{
Mesures de contrainte en médecine
}

\author{
Directives médico-éthiques de I'ASSM (1ère publication pour la procédure de consultation; la version allemande \\ est la version d'origine)
}

\section{Introduction}

Les mesures de contrainte représentent toujours une atteinte sévère au droit à la libre disposition de soi et au droit à la liberté personnelle d'un individu. Bien qu'en médecine, la non-contrainte soit un objectif prioritaire, il n'est pas toujours possible d'éviter la prise de mesures coercitives en ultime recours. Si le patient représente un danger grave pour lui-même ou pour autrui, de telles mesures constituent parfois la seule possibilité dont on dispose pour éviter des atteintes plus graves.

Les mesures de contrainte médicales [1] impliquent toujours un conflit entre plusieurs principes médico-éthiques: d'une part, il s'agit de «faire du bien» resp. de«ne pas nuire»; de l'autre, il s'agit de préserver autant que possible l'autonomie du patient. Par principe, tout acte médical présuppose le consentement du patient (informed consent). C'est pourquoi, les mesures de contrainte ne doivent être appliquées que dans des cas d'exception.

Dans des situations d'urgence où le patient fait courir un danger grave à lui-même ou à des tiers, la nécessité de prendre des mesures de contrainte n'est guère contestée. La question est plus difficile dans des situations ne présentant pas un caractère d'urgence, où les aspects de sécurité ou d'atteinte à la santé sont au premier plan, tout particulièrement en gériatrie et en psychiatrie. Ici, il est souvent difficile de déterminer clairement si la limitation des droits de l'individu et de sa liberté, c'est-à-dire la violation ponctuelle de l'autonomie du patient, afin de lui «faire du bien», justifie effectivement une mesure de contrainte.

En Suisse, il n'existe pas encore de bases légales uniformes relatives aux mesures de contrainte. Dès lors, les modalités des mesures de contrainte médicales sont diverses et dépendent des habitudes des différents établissements et des réglementations cantonales.

Les présentes directives doivent fournir une aide aux médecins et thérapeutes dans ce contexte difficile. Elles s'adressent à toute l'équipe soignante dans les établissements médicaux (hôpitaux et foyers), aux praticiens dans leur cabinet privé ainsi qu'au domaine des soins ambulatoires.
Elles abordent essentiellement les problèmes suivants:

- Dans quelles conditions éthiques et juridiques les mesures de contrainte sont-elles admises et justifiées?

- Quelles démarches convient-il d'entreprendre pour éviter les mesures de contrainte éventuellement envisagées?

- Comment informer les personnes concernées et, le cas échéant, leurs représentants thérapeutiques et légaux et leurs proches?

- De quelle manière peut-on mettre en œuvre les mesures de contrainte d'une façon aussi peu traumatisante que possible, lorsqu'elles se révèlent indispensables?

- Comment assurer le suivi des personnes ayant fait l'objet de mesures de contrainte?

- Comment documenter la procédure choisie?

\section{Champ d'application}

Les présentes directives se rapportent à toutes les situations thérapeutiques médicales pouvant se présenter dans le cadre de soins ambulatoires ou dans le cadre d'une hospitalisation. Les prises en charge socio-pédagogiques et non-médicales n'entrent pas dans le cadre de ces directives. Ces directives s'adressent non seulement au personnel médical, mais à toutes les personnes participant à la prise en charge d'un patient dans une situation thérapeutique médicale, sous réserve des dispositions cantonales ou fédérales.

\section{Principes}

\subsection{Définitions}

\subsubsection{Mesure de contrainte}

Par mesure de contrainte, on entend toute intervention allant à l'encontre de la volonté déclarée du patient ou suscitant sa résistance, ou, si le patient n'est pas capable de communiquer, allant à l'encontre de sa volonté présumée. Sont également considérées comme mesures de contrainte des mesures moins incisives comme p.ex. le fait d'obliger un patient à se lever, à manger ou à assister à une session thérapeutique. par mesures médicales, on entend soins. 
Dans la pratique, on peut distinguer entre l'entrave à la liberté et le traitement forcé.

3.1.1.1 Entrave à la liberté: On parle d'entrave à la liberté lorsqu'il s'agit exclusivement d'une restriction à la liberté de mouvement. Les entraves majeures à la liberté sont la privation de liberté à des fins d'assistance (cf. 4.3), la contention (p.ex. avec des sangles) ou l'isolement (p.ex. dans une chambre d'isolement).

Si l'entrave à la liberté intervient contre la volonté de la personne concernée, même si cette volonté n'est que présumée, elle doit être considérée comme une mesure de contrainte.

2 Ces droits, garantis par la Constitution comprennent selon celle-ci le droit à la liberté personnelle, en particulier à l'intégrité physique et psychique ainsi qu'à la liberté de mouvement. Les mesures de contrainte en tant qu'atteintes aux droits fondamentaux ne sont admises que si elles reposent sur une base légale et sont dans l'intérêt public prépondérant ou motivées par la protection de droits fondamentaux de tiers; en outre, elles doivent être proportionnées et ne pas porter atteinte à l'essence des droits fondamentaux. En principe, toutes ces conditions doivent être réunies pour qu'une mesure de contrainte puisse être mise en œuvre. Toutefois, dans de nombreux cantons, les bases légales font encore défaut.

3 En vertu de cette disposition légale, «une personne majeure ou interdite peut être placée ou retenue dans un établissement approprié lorsque, en raison de maladie mentale, de faiblesse d'esprit, d'alcoolisme, de toxicomanie ou de grave état d'abandon, l'assistance personnelle nécessaire ne peut lui être fournie d'une autre manière.»

4 Dans le cas de la privation de liberté à des fins d'assistance, c'est l'assistance qui se situe au premier plan; l'absence de capacité de discernement n'est donc pas une condition sine qua non. Par contre, un traitement ne peut être administré sous la contrainte qu'en cas d'absence de capacité de discernement concernant la nécessité d'un traitement.

5 D'après la loi, toutes les personnes de moins de 18 ans sont des «enfants» et des «mineurs». Dans le langage courant, toutefois, les enfants de plus de 11 ou 12 ans sont qualifiés d'adolescents. Dans ce paragraphe, nous utilisons le terme juridique «mineur» pour désigner toute personne de moins de 18 ans.
3.1.1.2 Traitement sous contrainte: Lorsqu'il y a non seulement entrave à la liberté, mais atteinte à l'intégrité physique d'une personne (p.ex. lorsqu'une médication est administrée sous la contrainte ou avec usage de la force), il s'agit d'une mesure de contrainte médicale avec atteinte à l'intégrité physique. On utilise alors le terme de «traitement sous contrainte».

Dans la pratique médicale quotidienne, les mesures de contrainte sont appliquées dans diverses disciplines et dans différentes situations. Les mesures de contrainte peuvent être prises dans des situations d'urgence psychosociale auquel cas il faut les distinguer selon qu'elles concernent des adultes, des enfants/adolescents ou des personnes âgées souffrant de troubles confusionnels - ou dans le domaine somatique.

\subsection{Cadre légal}

\subsubsection{Principe}

Les dispositions suivantes concernant le cadre légal se limitent pour l'essentiel au droit public. Les mesures de contrainte se situent dans un champ de tension juridique:

D'une part, les mesures de contrainte constituent toujours une atteinte aux droits fondamentaux du patient [2]. D'autre part, une injonction thérapeutique prononcée par l'Etat ou un devoir d'assistance peut être à l'origine d'une obligation légale de prendre une mesure de contrainte destinée à protéger le patient ou des tiers.

S'agissant en particulier des exigences légales, il faut distinguer entre le placement forcé dans un établissement avec, consécutivement, entrave à la liberté de mouvement et les autres mesures de contrainte médicales. En Suisse, une mesure de placement ne peut être prononcée que dans le cadre légal de la privation de liberté à des fins d'assistance, conformément à l'article 397 a du code civil (CC) [3] ou dans un autre cadre légal (p.ex. loi sur les épidémies). La décision de placement est prise par une autorité de tutelle du domicile. Pour les cas de péril en la demeure ou de maladie psychique, les cantons peuvent en outre attribuer cette compétence à d'autres offices appropriés (CC art. 397 b). De nombreux cantons ont attribué cette compétence de placement à des médecins de district, des médecins de santé publique ou des praticiens libéraux. Le placement devrait être décidé par des médecins suffisamment qualifiés ou spécialement formés. Le patient ou ses proches peut en appeler au juge pour qu'il statue sur la décision de placement (CC art. 397 d).

Les dispositions relatives à la privation de liberté à des fins d'assistance ne comportent aucune base légale pour les mesures de contrainte médicales au sens strict (traitements imposés) [4]. Néanmoins, de façon générale (voir conditions particulières dans le cas de mineurs, 3.2.2), ces mesures ne doivent être ordonnées que si une privation de liberté à des fins d'assistance a été décidée. Les situations d'urgence y font exception.

C'est la législation cantonale sur la santé qui détermine si des mesures de contrainte sont admises et à quelles conditions. Les dispositions légales cantonales en vigueur présentent cependant de fortes disparités de forme et de contenu. Cette situation hétérogène n'est pas satisfaisante du point de vue de la sécurité du droit. Une réglementation légale uniforme sur le plan suisse est d'autant plus nécessaire.

La participation des médecins à des mesures de contrainte policières ainsi que l'exercice de la médecine auprès de personnes détenues sont traités dans des directives séparées de l'ASSM (Directives médico-éthiques pour l'exercice de la médecine auprès de personnes détenues; 2002).

\subsubsection{Conditions particulières dans le cas de mineurs [5]}

Mineurs capables de discernement: En principe, les mineurs capables de discernement doivent donner leur accord à un traitement médical. Un mineur est capable de discernement lorsqu'il est capable de saisir la portée d'une intervention médicale et les conséquences d'un renoncement à une telle intervention et lorsqu'il dispose de la capacité d'exprimer sa volonté de son plein gré. Pour autant qu'ils le demandent, les adolescents capables de discernement doivent pouvoir prendre des décisions sans l'accord de leurs parents, dans la mesure où il ne s'agit pas de mesures consécutives à une mise en danger de soi et/ou d'autrui. En cas de refus, une mesure de contrainte n'est possible que si elle est dans 
l'intérêt supérieur du mineur. Dans ce cas, il faut procéder de la même manière que pour un adulte.

Mineurs incapables de discernement: Si le mineur est incapable de discernement, le droit de consentir à un traitement ou de le refuser revient au représentant légal (parents, tuteur).

Lorsque les parents ou le représentant légal prennent une décision contraire au bien de l'enfant, en dehors des situations d'urgence (p.ex. en cas de refus de consentir à une transfusion sanguine vitale), c'est l'autorité tutélaire qui est chargée de prendre une décision.

\subsection{Proportionnalité}

Les mesures de contrainte peuvent être extrêmement traumatisantes. Tout particulièrement dans le cadre des mesures de contrainte, le principe de proportionnalité doit être respecté; cela signifie qu'une telle mesure doit être premièrement nécessaire, deuxièmement proportionnelle au degré de danger encouru et troisièmement qu'elle ne peut être remplacée par des mesures moins radicales. Il faut donc, dans chaque cas, déterminer quelle est la mesure la moins lourde pour la personne concernée. De plus, il faut évaluer si le bénéfice (personnel et social) qu'on peut attendre de la mesure prime nettement sur l'atteinte qu'elle peut porter au patient ou si ses conséquences sont moins graves que celles d'une autre mesure qui, à défaut, s'imposerait. La durée de la mesure de contrainte doit également être adaptée aux circonstances. De plus, une mesure de contrainte doit être choisie sur la base des dernières connaissances disponibles et elle doit être réversible.

Lors de l'évaluation de la situation, il convient de tenir compte du fait qu'une mesure de contrainte peut entraîner des atteintes somatiques et psychiques. Il y a risque d'atteintes somatiques (p.ex. thromboses, infections) lors d'une immobilisation prolongée (p.ex. contention ou sédation) ou lors du recours à la force physique (p.ex. contusions, fractures). Une mesure de contrainte risque d'autant plus de provoquer des traumatismes psychiques que l'intervention est ressentie comme injustifiée ou humiliante, voire comme une rétorsion ou une atteinte délibérée.

\section{Processus décisionnels}

\subsection{Généralités}

En principe, toutes les dispositions possibles doivent être prises, permettant d'éviter des me- sures de contrainte, et avant qu'une mesure de contrainte ne soit engagée, toutes les autres options thérapeutiques moins radicales ayant des chances de succès doivent avoir été épuisées.

Les mesures de contrainte médicales impliquent une prescription médicale. Dans des situations d'urgence, des mesures limitatives de liberté peuvent aussi être mises en œuvre par des membres d'une équipe soignante, en particulier par le personnel soignant. Un consensus au sein de l'équipe soignante doit être recherché.

Dans toutes les institutions où des mesures de contrainte médicales sont mises en œuvre, les processus décisionnels doivent être stipulés par écrit et les postes responsables nommément désignés.

Si des situations d'urgence se répètent, chaque crise doit faire l'objet d'une évaluation spécifique. Tout particulièrement en milieu hospitalier, la prescription «à l'avance» d'un traitement sous contrainte est inadmissible. Dans de telles situations, une prévoyance adéquate permettrait d'éviter le recours à des mesures de contrainte.

\subsubsection{Information}

En principe, chaque traitement médical doit être précédé d'une information du patient, aussi complète et objective que possible. Cette information doit porter notamment sur le diagnostic, les examens et traitements prévus, les options thérapeutiques, les conséquences d'une absence de traitement, ainsi que sur les risques et effets secondaires. Ce devoir d'information incombant au médecin s'applique aussi aux mesures de contrainte. Des exceptions ne sont admises que s'il est nécessaire d'agir immédiatement et si le patient n'est manifestement pas en mesure de comprendre la mesure. Dans ce cas, l'information doit être fournie plus tard, dès que le patient a retrouvé sa capacité de discernement, s'il la retrouve. Les membres de la famille et les autres personnes de confiance proches du patient, désignés par lui, doivent être informés.

Si le patient dispose de voies de recours contre la prescription d'une mesure de contrainte, il doit en être informé également. Si, compte tenu de son état, le patient n'est pas en mesure de recevoir ou de comprendre cette information, elle doit lui être fournie plus tard et le plus tôt possible. En cas de privation de liberté à des fins d'assistance, c'est l'institution de placement qui est chargée d'informer le patient. Ses proches, qu'il a lui-même désignés, ainsi que d'autres personnes de référence qui lui sont proches doivent également en être informés. 


\subsubsection{Plan de traitement et consentement}

a) Soins ambulatoires: Dans de nombreuses situations d'urgence, le médecin en charge du patient ne peut pas déléguer la responsabilité d'un éventuel traitement forcé. Toutefois, en cas de privation de liberté à des fins d'assistance (cf. 4.3), le patient doit être informé qu'un placement forcé est la seule mesure raisonnable qui puisse être envisagée à ce moment-là. Le placement basé sur la privation de liberté à des fins d'assistance requérant des compétences et des expériences spécifiques, il est recommandé de déléguer cette tâche à des médecins spécialement formés. En cas d'impossibilité, le médecin concerné doit veiller à agir dans l'intérêt de son patient, même sous la pression de ses proches et de la police, et procéder soigneusement à tous les examens nécessaires. En situation d'urgence et si nécessaire, il a toutefois le droit d'administrer un médicament sans consentement, à condition que l'effet attendu soit dans l'intérêt du patient.

b) En milieu hospitalier: Les mesures de contrainte mises en œuvre sur une période prolongée en dehors d'une situation d'urgence doivent être intégrées à un plan de traitement global, l'absence de capacité de discernement étant la condition sine qua non. Dans ces cas, le consentement des représentants thérapeutiques [6] qu'il a lui-même désignés ou le consentement de son représentant légal doit être recherché. S'il existe des directives anticipées rédigées par le patient alors qu'il était capable de discernement, il faut en tenir compte dans le cadre d'éventuelles dispositions légales et des possibilités données. Pour la mise en œuvre de traitements de contrainte prévisibles dans des établissements hospitaliers, en plus de l'hospitalisation par un médecin, le consentement du directeur médical resp. de son adjoint est obligatoire. Le médecin en charge du patient peut décider uniquement en situation d'urgence. La responsabilité des mesures concernant des activités quotidiennes comme le fait d'obliger un patient à se lever, s'habiller etc., pouvant revêtir un caractère contraignant, est de la compétence des soignants concernés.

\subsubsection{Processus décisionnels dans le cas de mineurs}

Les décisions relatives au traitement et à la prise en charge de mineurs doivent être prises d'entente avec les parents ou les représentants légaux et dans le meilleur intérêt de l'enfant ou de l'adolescent.

Il est du devoir des professionnels d'associer autant que possible le mineur aux décisions et de solliciter son consentement en fonction de sa capacité de discernement, car le principe de libre disposition de soi vaut aussi pour les mineurs capables de discernement. Le fait d'associer le mineur améliore la communication entre les professionnels et les membres de la famille; de plus, les enfants et adolescents associés aux décisions coopèrent mieux au traitement. Toutefois, face à des personnes disposant d'une autorité, le consentement du mineur risque de ne pas être véritablement spontané.

Lors des prises de décisions, il ne faut pas exiger trop de l'enfant ou de l'adolescent; à l'inverse, il ne faut pas non plus décider à sa place lorsqu'il a le désir et la capacité de contribuer activement aux décisions relatives au traitement. La libre disposition de soi est un droit humain qui - moyennant adaptation appropriée - vaut aussi pour les enfants et adolescents. Tout comme la prise en charge de soi-même, le droit de libre disposition de soi s'exerce de manière progressive au cours de l'enfance et de l'adolescence, tout d'abord de manière limitée, puis de plus en plus pleinement jusqu'à la maturité.

Si un mineur ne consent pas à un traitement qu'on lui propose et qu'il comprend, ce traitement ne doit pas lui être prodigué. Si le mineur est considéré comme incapable de discernement dans la situation concrète et que la mesure est nécessaire parce qu'il fait courir un danger à lui-même et/ou à autrui, ses parents peuvent consentir à sa place à un traitement. En cas d'opposition des parents, l'autorité tutélaire doit examiner l'opportunité de mettre en place des mesures de protection de l'enfant.

\subsection{Situations problématiques particulières}

\subsubsection{Dans le domaine somatique}

4.2.1.1 Dans des situations d'urgence

- Lorsque des patients ont besoin de soins médicaux aigus et lorsque le pronostic vital est engagé, mais qu'ils refusent un traitement en raison d'un état de choc ou d'agitation psycho-motrice, on peut admettre qu'ils sont momentanément incapables de discernement. Il faut alors décider du traitement en fonction de leur volonté présumée.

- Lorsqu'une assistance médicale urgente est prodiguée après une tentative de suicide, alors que le patient ne formule aucune demande d'assistance médicale mais que celleci est d'une importance vitale, il faut, en règle générale, là aussi admettre que le patient est momentanément incapable de discernement (p.ex. en raison d'un état dépressif). 
4.2.1.2 Dans des situations subaiguës: Lors de maladies contagieuses à déclaration obligatoire, la loi sur les épidémies prévoit que les autorités sanitaires peuvent imposer des mesures (isolement, médication forcée), s'il le faut même contre la volonté du patient. Cependant, dans une telle situation également, il faut tout faire pour que le patient accepte de son plein gré les mesures thérapeutiques. Il en va de même, par analogie, pour les infections nosocomiales (contractées à l'hôpital).

\subsubsection{Dans des situations d'urgence psychosociale} 4.2.2.1 Généralités: Les troubles psychiques graves peuvent conduire à une perte de contrôle et à des comportements faisant courir un danger aigu à la personne concernée ou à des tiers. C'est uniquement dans des cas de ce genre que des mesures de contrainte peuvent être mises en œuvre.

Si la situation d'urgence psychosociale ne peut être résolue d'une autre manière, une privation de liberté à des fins d'assistance peut être envisagée (voir le chapitre 3.3). Dans le cadre d'une privation de liberté à des fins d'assistance, d'autres mesures ne découlant pas purement de cette nécessité d'assistance, comme l'isolement, la contention et la médication forcée, peuvent être nécessaires dans certaines circonstances. Parmi les motifs de mise à l'isolement figurent la perte momentanée du contrôle de soi avec agitation violente manifeste, la menace de violence caractérisée ou la mise en danger d'autrui, ainsi que la perturbation grave de la vie en communauté. Les motifs de contention peuvent être par exemple des tentatives graves d'automutilation resp. un danger aigu d'automutilation. Une médication forcée peut se révéler indispensable lors d'états aigus d'excitation avec mise en danger du patient lui-même ou d'autrui, ou pour éviter un isolement ou une contention répétés ou prolongés. En principe, l'isolement et la contention ne devraient pas durer plus de quelques heures.

Dans des situations d'urgence psychosociale, il faut tout d'abord essayer, selon un plan progressif, d'autres possibilités de désescalade, pour autant qu'il n'y ait pas de danger imminent. En milieu hospitalier, il s'agit en particulier de la désescalade verbale («talking down»), de la fixation verbale de limites, de l'isolement dans la propre chambre, de la stimulation au mouvement ou de la présentation d'autres solutions possibles. Il faut aussi examiner l'opportunité d'une prise en charge individuelle sur une période prolongée avec accompagnement constant, dans la mesure où la sécurité de la personne effectuant cette prise en charge peut être garantie.
Etant donné la diversité des situations d'urgence psychosociale, certaines conditions et procédures particulières doivent en outre être observées:

4.2.2.2 Mise en danger de soi: Il y a mise en danger de soi lorsque le comportement pathologique menace de causer des atteintes immédiates au patient lui-même. Cela impose, quel que soit le type de mesure de contrainte, de déterminer soigneusement la proportionnalité; en particulier, il faut toujours examiner la faisabilité d'une prise en charge individuelle. Les mesures de contrainte motivées par une mise en danger de soi ne sont donc admises qu'en cas d'incapacité de discernement ou dans des formes particulières de perte de contrôle (p.ex. danger imminent pour l'intégrité physique et la vie en cas de comportement suicidaire ou d'automutilation).

4.2.2.3 Mise en danger d'autrui: Il y a mise en danger d'autrui lorsqu'il y a danger prévisible pour d'autres personnes que le patient lui-même. Un danger existe notamment en cas de comportement agressif pouvant aller jusqu'à la menace de violence grave ou d'attaques physiques. Une intervention médicale allant contre la volonté de la personne concernée suppose que la cause du comportement dangereux pour autrui réside dans un trouble psychique et que le danger est imminent.

4.2.2.4 Perturbation grave de la vie en communauté: Il y a perturbation grave de la vie en communauté lorsque le comportement d'un patient souffrant d'une maladie psychique est à ce point éprouvant pour son entourage immédiat ou que celui-ci porte atteinte à la liberté des autres de telle façon qu'une vie en communauté avec lui n'est plus possible [7]. Les mesures de contrainte, en particulier une privation de liberté à des fins d'assistance, ne se justifient dans un tel cas que si le comportement éprouvant du patient est imputable à une maladie et nécessite un traitement, et que d'autres mesures librement consenties ont échoué.

\subsubsection{Etats confusionnels des personnes âgées}

Lors de la prise en charge par exemple de patients déments, des mesures entravant la liberté sont souvent inévitables en cas de confusion et d'agressivité. L'Académie Suisse des Sciences Médicales a élaboré des directives spécifiques sur le traitement et la prise en charge des personnes âgées en situation de dépendance et, dans ce cadre, a émis des recommandations quant aux mesures limitatives de liberté éventuellement 
rendues nécessaires chez des personnes souffrant de troubles confusionnels. En voici la teneur:

«Sauf disposition légale contraire, une mesure limitative de liberté ne peut être prise qu'aux conditions suivantes:

a) par son comportement, la personne âgée compromet gravement sa sécurité, sa santé ou celles de tiers, ou perturbe gravement la paix et le bien-être de ceux-ci;

b) le comportement particulier n'est pas dû à des causes auxquelles il est possible de remédier (douleurs, effets secondaires de médicaments ou tensions interindividuelles, p.ex.);

c) d'autres mesures moins restrictives de la liberté personnelle ont échoué ou ne peuvent pas être appliquées.

Avant d'être proposée à la personne âgée ou, en cas d'incapacité de discernement, à son représentant thérapeutique ou à son représentant légal, une mesure limitative de liberté doit avoir fait l'objet d'une discussion entre le médecin et l'équipe soignante et les thérapeutes.

La personne âgée ou, en cas d'incapacité de discernement, son représentant thérapeutique ou son représentant légal, doit être informée, de manière claire et appropriée, du but, de la nature et de la durée de la mesure limitative de liberté; le nom de la personne responsable doit également lui être communiqué.

Une mesure limitative de liberté ne peut être prise qu'avec l'assentiment de la personne âgée concernée ou, si elle est incapable de discernement, avec le consentement de son représentant thérapeutique resp. de son représentant légal.

Si une personne incapable de discernement n'a ni représentant thérapeutique, ni représentant légal, ou lorsque l'urgence ne permet pas de les contacter, le médecin, les soignants et, le cas échéant, les thérapeutes concernés prennent la décision de manière interdisciplinaire, en incluant les proches dans le processus décisionnel. Ils agissent conformément aux intérêts objectifs et à la volonté présumée de la personne concernée en respectant les conditions susmentionnées [8]. Les décisions immédiates prises par une seule personne doivent être réévaluées ensuite selon ce même processus.»

\subsection{Privation de liberté à des fins d'assistance}

Sous réserve de dispositions légales contraires, exigeant par exemple la désignation et l'assentiment d'un représentant thérapeutique ou d'un représentant légal.

\subsubsection{Généralités}

Du fait de leur état, les personnes souffrant d'une maladie psychique et placées dans un établisse- ment psychiatrique dans le cadre d'une privation de liberté à des fins d'assistance (cf. 3.2, Cadre légal), ne sont souvent pas capables de discernement et de consentement, ou ne le sont que partiellement. Toutefois, le fait qu'elles soient placées dans un établissement ne justifie pas en soi n'importe quelle mesure médicale (ni n'importe quelle autre mesure entravant davantage la liberté personnelle au sein de l'établissement) allant contre la volonté du patient. Par principe, les personnes placées en vertu d'une privation de liberté à des fins d'assistance disposent elles aussi de tous les droits revenant aux patients, c'est-àdire, notamment, du droit d'être informé de manière complète sur le tableau clinique, les possibilités de traitement et les risques et effets secondaires, ainsi que sur les conséquences qu'aurait l'absence de traitement. En règle générale, le consentement des patients placés est également nécessaire pour toute mesure diagnostique, préventive ou thérapeutique. Dans le cadre d'un traitement d'urgence, on ne peut faire abstraction de ce consentement que si une intervention immédiate s'impose pour écarter un danger imminent d'atteinte grave à la santé ou de décès. Si une mesure médicale allant contre la volonté du patient se révèle indispensable, les dispositions légales doivent être respectées. Les mesures de contrainte prises en vertu d'une privation de liberté à des fins d'assistance ne doivent être mises en œuvre que tant que la situation qui les a motivées (mise en danger de soi ou d'autrui, ou perturbation grave de la vie en communauté au sein de l'établissement) persiste.

\subsubsection{Privation de liberté à des fins d'assistance dans le cas de mineurs}

Une privation de liberté à des fins d'assistance touchant un mineur constitue en fait un retrait du droit de garde par l'autorité de tutelle. La prise en charge est exécutée dans un établissement (foyer, clinique, service de surveillance médicale). Les dispositions relatives à la privation de liberté à des fins d'assistance de personnes adultes s'appliquent par analogie à la procédure et au recours judiciaire. Dès 16 ans, un adolescent peut lui-même recourir au juge et demander en tout temps la levée de la mesure (CC art. 314 a). Les autorités légales compétentes pour décider du placement doivent garantir si possible une permanence 24 heures sur 24, y compris les dimanches et jours fériés. Dans la plupart des cantons, des médecins praticiens sont également habilités à décider du placement d'un mineur souffrant d'une maladie psychique.

L'hébergement des mineurs devrait être séparé de celui des adultes. 


\section{Mise en œuvre}

\subsection{Principe}

Toute mesure de contrainte doit suivre une stratégie d'action claire et précise. Une fois la décision prise, une démarche ciblée, coordonnée et résolue, décidée au sein de l'équipe soignante, et incluant toutes les personnes concernées, en milieu hospitalier est indispensable. Toute agressivité - y compris verbale - inappropriée doit être évitée. Egalement dans le cadre des soins ambulatoires, toutes les mesures pouvant contribuer à la désescalade de la situation doivent être prises.

Ne sont en principe autorisées que les mesures qui répondent aux normes reconnues de la spécialité médicale concernée.

Sont interdits les actes inutilement douloureux ou entravant la liberté personnelle, notamment la liberté de mouvement, dans une mesure supérieure à ce qui est absolument nécessaire. Des mesures de contrainte ne peuvent pas être engagées pour discipliner ou punir des patients.

\subsection{Aspects particuliers}

Lors de la mise en œuvre de mesures de contrainte, il importe de veiller tout spécialement aux aspects suivants:

- Il faut créer un environnement aussi sûr que possible; les objets susceptibles de mettre en danger le patient ou le personnel doivent être enlevés. Le lieu de mise en œuvre de la mesure doit être choisi avec soin.

La sphère intime du patient doit être préservée, la présence de personne non concernées est à éviter.

Le début de la mise en œuvre de la mesure de contrainte est communiqué de manière claire et compréhensible au patient. Il est bon qu'une seule personne dirige le déroulement de l'acte. Un comportement hésitant ou des instructions contradictoires dans la mise en œuvre de la mesure de contrainte compliquent le déroulement de l'acte et stressent le patient.

L'acte de mise en œuvre d'une mesure de contrainte doit se dérouler aussi calmement que possible.

Les différentes phases de l'acte doivent être annoncées de manière claire et compréhensible.

L'aspect imposant du déploiement en personnel freine dans bien des cas l'agressivité du patient.

Avant l'administration d'une éventuelle injection forcée, il est recommandé de suivre la procédure suivante: la médication doit être encore une fois proposée par voie buccale, en sachant bien que cette méthode représente déjà une mesure de contrainte. Ce n'est que si le patient refuse à nouveau l'ingestion de la médication que celle-ci est administrée contre son gré par voie parentérale.

\subsubsection{Durée}

La durée de toute mesure de contrainte, en particulier en milieu hospitalier, doit être limitée dès le début. Il faut déterminer, dès la prescription, quand le prochain réexamen de la mesure devra être entrepris. Ce réexamen devrait avoir lieu le plus souvent possible, par exemple toutes les heures en cas d'isolement ou de contention. En règle générale, les mesures de contrainte ne doivent être mises en ouvre que tant qu'elles sont absolument indispensables.

\subsubsection{Surveillance en milieu hospitalier}

La personne concernée par la mesure de contrainte fait l'objet d'une prise en charge continuelle et d'une surveillance adaptée à la situation.

En fonction de la mesure et de l'état du patient, des mesures prophylactiques appropriées doivent être prises (prévention d'une pneumonie, des escarres de décubitus, etc.).

\subsubsection{Accompagnement et suivi}

Les mesures de contrainte sont vécues par les personnes concernées comme des événements particulièrement pénibles, pouvant, de surcroît, entraîner des conséquences physiques et psychiques. Pour cette raison également, la situation générale doit être réévaluée régulièrement, de manière à ce que la mesure de contrainte ne dure que le temps absolument nécessaire.

Toute mesure de contrainte médicale - aussi bien dans le domaine des soins ambulatoires qu'en milieu hospitalier - appelle une discussion ultérieure avec le patient dès que son état le permet. S'il le souhaite, d'autres personnes peuvent être associées à cette discussion. A cette occasion, les médecins responsables doivent encore une fois exposer les raisons qui, de leur point de vue, on rendu la mesure nécessaire. Il faut accorder au patient un laps de temps suffisant pour exposer son vécu et son point de vue. Il faut en outre lui donner la possibilité d'exprimer par écrit son vécu en vue de l'anamnèse. Le risque de traumatisme psychique est d'autant plus élevé que l'intervention a été ressentie comme injustifiée ou humiliante, voire comme une atteinte délibérée.

Dès lors, il faut chercher à savoir si la mesure de contrainte a provoqué une atteinte psychique nécessitant éventuellement une thérapie spécifi- 
que. Les mesures de contrainte appliquées devraient également être discutées par la suite au sein de l'équipe soignante.

\subsubsection{Documentation}

Toute mesure de contrainte doit être soigneusement documentée dans le dossier médical et le dossier infirmier du patient. Il importe de consigner au moins les points suivants: motifs et base légale éventuelle de la mesure, type, durée et moment de la contrainte exercée, personnes responsables de la prescription et de la mise en œuvre, contrôles effectués, information donnée.

Dans l'ordonnance d'une privation de liberté à des fins d'assistance, la documentation doit contenir au moins l'heure précise de l'examen médical, l'indication du type de trouble psychique diagnostiqué, l'heure précise et l'endroit du placement, une brève justification de la privation de liberté à des fins d'assistance, l'indication du droit d'audience. Un exemplaire de l'ordonnance doit être remis au patient.

\subsubsection{Voies de recours}

L'accès aux possibilités légales d'opposition et de recours doit être garanti.

Avant la prescription d'une mesure de contrainte prévue, le patient doit être rendu attentif, si possible par écrit, aux dispositions légales applicables et aux possibilités concrètes de recours dont il dispose (voir 3.1.1).

\section{Encadrement en personnel et institutionnel}

Dans chaque établissement il est indispensable que soient réunies les conditions en personnel et institutionnelles lui permettant de renoncer autant que possible aux mesures de contrainte. Les insuffisances institutionnelles et en ressources humaines ont une influence déterminante sur les décisions relatives aux mesures de contrainte, et elles ne doivent pas les légitimer.

Les délinquants souffrant de maladies psychiques doivent être pris en charge par des institutions spécialisées.
L'institution veille à ce que ses collaborateurs aient connaissance des bases juridiques et des directives; elle définit clairement et par écrit les processus décisionnels et les responsabilités.

Elle met à disposition un effectif de personnel formé suffisant à la mise en pratique des présentes directives.

Des formations et supervisions sur le thème de la désescalade et de la gestion des mesures de contrainte et de la violence doivent être proposées.

\section{Membres de la sous-commission}

Prof. Daniel Hell, Zurich, président; Prof. HansDieter Brenner, Berne; Prof. Volker Dittmann, Bâle; Dr Carlo Foppa, Lausanne; Dr Jürg Gassmann, Zurich; Dr Christian Hess, Affoltern; Dr Margrit Leuthold, Bâle, ex officio; Dr Agnès Michon, Genève; Dr Eberhard Rust, Oberwil b. Zug; Renata Schläpfer, Reussbühl; PD Dr Barbara Steck, Bâle; Urs Trottmann, Zurich; Prof. Michel Vallotton, Genève, Président CCE, ex officio.

Nos remerciements vont aux experts ci-après pour leurs précieux conseils

Prof. Peter Aebersold, Bâle; Dr Giusep Nay, Lausanne; Prof. Andreas Stuck, Berne; Urs Vogel, Lucerne.

Directives de I'ASSM citées

- «Directives médico-éthiques concernant l'exercice de la médecine auprès de personnes détenues»; 2002.

- «Directives médico-éthiques sur le traitement et la prise en charge des personnes âgées en situation de dépendance»; 2004.

Autres réglementations significatives

- Convention relative à la bioéthique (pas encore ratifiée par la Suisse). www.ofj.admin.ch/f/ index.html.

- Convention relative aux droits de l'enfant. Hautcommissariat aux droits de l'homme. www. unhchr.ch/french/html/menu3/b/k2crc_fr.htm.

- Projet de la nouvelle loi sur la protection des adultes. www.ofj.admin.ch/f/index.html.

- Principles for Policy on Mental Health (UNO). 